\title{
Enfermería de Práctica Avanzada para el fortalecimiento de la atención primaria de salud en el contexto de Latinoamérica ${ }^{1}$
}

Elena Bustos Alfaro ${ }^{2}$

Institución: Universidad Federal de Río Grande-FURG-

\section{ENSAYO}

\section{RESUMEN}

Este trabajo pretende mostrar el papel significativo que puede llegar a desarrollar la Enfermería de Práctica Avanzada (EPA) en el fortalecimiento de la atención primaria de salud (APS), así como la situación de este fenómeno en los países de Latinoamérica. La evidencia demuestra que las EPA en la APS brindan atención de alta calidad y rentable para los sistemas de salud de los países, incluso existen resultados con los pacientes que son comparables o mejores que las contrapartes médicas. Se conoce que actualmente la OPS/OMS está estableciendo un plan de trabajo para apoyar la ampliación y profesionalización de la EPA, cuya prioridad es la preparación y profesionalización de enfermeras para que fomenten de forma efectiva la implementación de la Cobertura Universal de Salud.

Palabras claves: Atención-primaria-de-salud; Enfermería-de-práctica-avanzada; Recursos-humanos.

DOI: $10.15517 /$ revenf.v0iNo. 37.34645

${ }^{1}$ Fecha de recepción: 24 de setiembre del 2018

Fecha de aceptación: 09 de abril del 2019

${ }^{2}$ Enfermera, Máster en Práctica Avanzada y Gestión de Enfermería, Doctoranda en Enfermería por Universidad Federal de Río GrandeFURG- Brasil. Correo electrónico: ebustos@alumni.unav.es 


\title{
Advanced Practice Nursing for the strengthening of primary health care in the context of Latin America ${ }^{1}$
}

Elena Bustos Alfaro ${ }^{2}$

Institution: Federal University of Rio Grande-FURG

\section{ESSAY}

\begin{abstract}
This paper aims to show the significant role that Advanced Practice Nursing (APS) can play in the strengthening of Primary Health Care (PHC), and what is the situation of this phenomenon in Latin American countries. The evidence shows that the EPAs in the APS, provide high quality and cost-effective care for the health systems of the countries, there are even results with patients that are comparable or better than the medical counterparts. It is known that PAHO/WHO is currently establishing a work plan to support the expansion and professionalization of the EPA, prioritizing the preparation and professionalization of nurses so that they are prepared to effectively promote and implement Universal Health Coverage.
\end{abstract}

Keywords: Advanced-practice-nursing; Human-resources; Primary-health-care.

DOI: $10.15517 /$ revenf.v0iNo. 37.34645

${ }^{1}$ Date of receipt: September 24, 2018

Date of acceptance: April 09, 2019

2 Nurse, Master's Degree in Advanced Practice and Nursing Management, PhD student in Nursing, Federal University of Rio Grande-

FURG-Brazil. E-mail: ebustos@,alumni.unav.es 


\title{
Enfermagem de Prática Avançada para o fortalecimento da atenção primária em saúde no contexto da América Latina ${ }^{1}$
}

\author{
Elena Bustos Alfaro²
}

Institução: Universidade Federal do Rio Grande

\section{ENSAIO}

RESUMO

Este trabalho tem como objetivo mostrar o papel significativo que a Enfermagem de Prática Avançada (EPA) pode desempenhar no fortalecimento da Atenção Primária à Saúde (APS) e qual é a situação desse fenômeno nos países da América Latina. As evidências mostram que as EPAs na APS proporcionam cuidados de alta qualidade e benéfico para os sistemas de saúde dos países, tendo resultados com os pacientes comparados e/o melhores que os colegas médicos. Sabe-se que a OPS/OMS estão atualmente estabelecendo um plano de trabalho para apoiar a expansão e profissionalização da EPA, priorizando a preparação e profissionalização dos enfermeiros para que estejam preparados para promover e implementar efetivamente a Cobertura Universal de Saúde.

Palavras-chave: Atenção-primária-à-saúde; Enfermagem-de-prática-avançada; Recursos-humanos.

DOI: $10.15517 /$ revenf.v0iNo. 37.34645

${ }^{1}$ Data de recepção: 24 de setembro de 2018

Data de aceitação: 9 de abril de 2019

${ }^{2}$ Enfermeira, Mestrado em Prática Avançada e Gestão em Enfermagem, Doutoranda em Enfermagem pela Universidade Federal do Rio Grande - FURG- Brasil. Correio eletrônico: ebustos@alumni.unav.es 


\section{INTRODUCCIÓN}

Las enfermeras son fundamentales para los sistemas de salud, dado que prestan la mayor parte de los servicios de atención ${ }^{1}$, por ello en un sistema de salud tan cambiante como el actual, es cada vez más imperante dirigir continuamente la práctica de enfermería a los beneficios de la población, necesidad que ha sido un fenómeno global, motivo por el que se debate en muchos países.

Tanto la profesión de Enfermería, como su ámbito práctico, son dinámicos porque se adaptan teórica y científicamente a las necesidades actuales de la sociedad, en torno a las que los profesionales en enfermería se constituyen en la médula espinal del sistema de cuidados de la salud ${ }^{2}$.

Aun así, en América Latina hay una escasez grave de personal de enfermería capacitado del que, al mismo tiempo, en ciertas ocasiones, no se obtiene el provecho que puede aportar al sistema de salud y a la población en general. En relación con lo anterior, expertos afirman que se debe aumentar el número de enfermeras mas, de igual forma, se debe mejorar la educación y ejercicio de la profesión para que este personal pueda desarrollar y utilizar plenamente sus aptitudes, conocimientos y experiencias ${ }^{1}$.

En América Latina no solo existe el problema de la formación de los profesionales de enfermería, sino que hay diferencias en acceso a la asistencia sanitaria de alta calidad y una carga de enfermedades transmisibles y enfermedades no transmisibles, las cuales generan desafíos en lo concerniente a una efectiva asignación y aplicación de recursos de salud ${ }^{3}$.

Desde esa perspectiva, la Atención Primaria de la Salud (APS) representa el núcleo del sistema de salud de un país, por lo que la presencia de los profesionales en este nivel es de suma relevancia, así como la labor que puede desarrollar el profesional en este ámbito, lo cual influirá en todos los demás niveles, debido a que la APS es toda aquella asistencia sanitaria esencial accesible a todos los individuos y familias de la comunidad, a través de medios aceptables, con una plena participación y a un costo asequible para la comunidad y el país ${ }^{4}$.

Por lo anterior es que las organizaciones a nivel mundial han planteado que la EPA puede ayudar al fortalecimiento de la APS, de lo cual se deriva la inquietud de cómo ha sido planteada esta necesidad para el contexto latinoamericano.

\section{DESARROLLO}

\section{La Enfermería de Práctica Avanzada (EPA) en la APS}

Primeramente, es importante aclarar que la APS es la puerta de entrada del sistema de salud y el lugar donde se operativiza la continuidad de la atención para la mayor parte de la población ${ }^{4}$. Se conoce que los países luchan por alcanzar este acceso y cobertura universal de la salud, por medio de un reconocimiento de los recursos humanos idóneos, entre ellos el profesional de enfermería; no obstante, por las condiciones de salud y asistencia sanitaria que presenta América Latina, la situación se torna compleja, motivo por el que se requiere un proceso de 
cambio y fomentar un rol más amplio de enfermería en Latinoamérica, cambio que involucra la voluntad política, lo cual implica un apoyo multifacético de los funcionarios de gobierno, legisladores, jefes de hospitales, personal médico y docentes de enfermería ${ }^{3}$, y un cambio de perspectiva por parte de los profesionales de enfermería.

En el pasado, lo que ha acontecido es que la mayor parte del personal de enfermería de América Latina y el Caribe corresponde a enfermeras licenciadas o tituladas (programas de grado que generalmente duran cuatro años) o son auxiliares o técnicos en enfermería (programas técnicos de menor duración). Quienes obtienen un título de maestría en enfermería, por lo general, terminan dando clases o en cargos gerenciales, no en los servicios de atención directa1, como lo es el primer nivel de atención.

En medio de todos estos cambios que presenta el sistema de salud, en otros países (USA, Canadá, en ciertos países de Europa) surge la Enfermería de Práctica Avanzada, debido a que se necesitaba de una reestructuración del cuidado de la salud, respecto de la formación de los profesionales de enfermería para que pudieran dar respuesta a las necesidades del sistema de salud.

En relación con lo mencionado, el papel que puede llegar a desarrollar la Enfermera de Práctica Avanzada (EPA) en el sistema de salud es significativo y aún más en la APS, motivo por el quepaíses como los Estados Unidos y Canadá han incorporado de manera activa el papel de la EPA dentro de sus sistemas de atención en salud para brindar APS para sus poblaciones, con énfasis en las comunidades más marginadas. En la actualidad, más de 205000 EPA están acreditadas en los Estados Unidos, de los cuales dos terceras partes ejercen en APS ${ }^{5}$.

La evidencia señala que la EPA puede contribuir en cuanto a que se avance a la estrategia de salud que plantea la APS; sin embargo, en nuestro ámbito es poco conocido el rol de este perfil profesional; por lo tanto, es importante definir este rol de enfermería: según el Consejo Internacional de Enfermería (ICN) (2008), la EPA es una enfermera registrada que ha adquirido la base de conocimiento experto, preferiblemente con maestría como nivel de entrada ${ }^{6}$, habilidades de toma de decisiones complejas y competencias clínicas para la práctica ampliada, cuyas características son moldeadas por el contexto o país en el que se le acredita para practicar.

Cabe resaltar que anteriormente cuando no existía este rol de la EPA, las enfermeras cumplían un papel clave de acuerdo con su conocimiento al brindar servicios en APS en áreas urbanas, rurales y marginadas. En décadas recientes, quienes han buscado formalizar este papel en algunos países han instado a los hospitales, las universidades y los legisladores de las políticas para apoyar de manera formal a los programas de EPA reconocidos para la $\mathrm{APS}^{5}$.

\section{Competencias que desarrolla la Enfermería de Práctica Avanzada (EPA)}

La Enfermería de Práctica Avanzada (EPA) puede contribuir a mejorar la APS, mediante una atención completa e integral de los pacientes, familias y comunidades. La EPA actúa a través de competencias profesionales, entre las que se destaca 7

Llevar a cabo una evaluación integral y sistemática de los parámetros de salud y enfermedad en situaciones complejas, incorporando enfoques diversos y culturalmente sensibles. 
- Diseñar, implementar y evaluar intervenciones terapéuticas basadas en la ciencia de enfermería y otras ciencias.

- Desarrollar y mantener relaciones terapéuticas y asociaciones con pacientes (individuos, familia o grupo) y otros profesionales para facilitar una atención y resultados óptimos.

- Demostrar niveles avanzados de pensamiento de sistemas de juicio clínico y rendición de cuentas al diseñar, distribuir y evaluar la atención basada en la evidencia para mejorar los resultados de los pacientes.

- Guía, asesora y brinda apoyo a otras enfermeras para alcanzar la excelencia en la práctica de la enfermería.

- Educa y guía a individuos y grupos a través de las complejas transiciones en salud.

- Usa las habilidades conceptuales y analíticas para evaluar los vínculos entre las cuestiones de la práctica, lo organizacional, de la población, así como a nivel de las políticas de cada país.

Existen diversas aproximaciones teóricas sobre la práctica avanzada: uno de los que ha tenido mayor impacto es el modelo de Hamric y sus colaboradoras (plantea siete competencias: práctica clínica directa, consultor, habilidades en investigación, liderazgo profesional y clínico, colaboración, habilidades en toma de decisiones éticas y coaching experto), el cual es un modelo teórico-conceptual, en el que han conceptualizado la práctica avanzada no solo a través de las competencias propias, denominadas nucleares, sino también desde los prerrequisitos o criterios primarios necesarios e imprescindibles para realizar la práctica avanzada, sin dejar de lado una serie de elementos del entorno a nivel de políticas de salud y de las organizaciones que influyen en el desarrollo de la $\mathrm{EPA}^{8-9}$.

Cabe resaltar que los contextos en los que una EPA puede trabajar son idénticos a los de cualquier enfermera asistencial. Sin embargo, el valor añadido de la Práctica Avanzada en Enfermería reside en los niveles de autonomía que desarrolla, la complejidad de su conocimiento y la toma de decisiones, el juicio clínico avanzado, y las habilidades en la gestión e innovación de organizaciones y de los servicios a un nivel superior al alcanzado en la enfermería genera ${ }^{2}$.

Debido a lo anterior, la evidencia demuestra que las EPA en la APS brindan atención de alta calidad y rentable, con mejores resultados en los pacientes o, al menos, comparables respecto de sus contrapartes médicas ${ }^{10}$. Sin embargo, el rol de la EPA en los países de Latinoamérica aún no ha sido bien establecido o reconocido ${ }^{5}$.

\section{Enfermería de Práctica Avanzada (EPA) en Latinoamérica}

En el 2015, cuando la OPS en conjunto con la Escuela de Enfermería y el Centro Colaborador de la Organización Mundial de la Salud (OMS) en Atención Primaria de Salud y Recursos Humanos de Salud, organizaron una cumbre sobre la Enfermería de Práctica Avanzada en la Universidad McMaster en Hamilton, Ontario, Canadá.

Los participantes en la cumbre de 2015 incluyeron líderes de enfermería de 17 países de las Américas, entre los que se encontraban representantes de los Ministerios de Salud, asesores de la OPS y de la OMS, decanos 
de Escuelas de Enfermería, presidentes de asociaciones de enfermería e investigadores de enfermería. Los objetivos generales de la cumbre fueron definir el alcance de los roles de enfermería, incluidos los de las EPA, así como discutir el contexto y las experiencias de los países de las Américas, desarrollar estrategias para la implementación y considerar el impacto que las funciones de EPA podrían tener para mejorar la salud ${ }^{11}$.

La implementación efectiva de los roles de APN en la APS en América Latina y el Caribe podría contribuir con la salud universal, principalmente en las comunidades rurales y remotas y otros grupos vulnerables de la población que luchan con el acceso limitado a los servicios básicos de salud. Esta brecha en la atención de la salud se ampliará a medida que aumenten el tamaño de la población y la prevalencia de enfermedades crónicas. La expansión de la APS integral para abordar las necesidades de salud de las poblaciones desatendidas requerirá la capacitación de recursos humanos adicionales de alta calidad para la salud ${ }^{11}$.

Con la resolución de la OPS se promueve más EPA para entregar APS, por lo que la OPS y otras organizaciones internacionales y asociaciones han redoblado esfuerzos para establecer, promover, implementar y reconocer el papel de las EPA en la región ${ }^{5}$.

En algunos países de América Latina y el Caribe existen programas de posgrado en enfermería, pero la mayoría se centran en la investigación o la educación y no en la práctica clínica avanzada ${ }^{12}$. En Costa Rica existen programas de maestría en los que se brinda la especialización para áreas de enfermería; se han implementado másteres profesionales en ginecología, obstetricia y perinatología, enfermería pediátrica con énfasis en neonatología o niñez y adolescencia, enfermería de salud mental, enfermería quirúrgica, entre otros. Sin embargo, en la atención primaria no parece expandirse la formación que alcance la APS.

Al respecto, países como Brasil, México, Colombia y Chile han iniciado sus propias discusiones para explorar la viabilidad de introducir el papel de la EPA en sus modelos nacionales de atención en salud ${ }^{13}$. En el caso de Chile, la Asociación Chilena de Educación en Enfermería, junto con asociaciones del gremio y la OPS, están avanzado en implementar el rol de la EPA en la APS, para lo que están diseñando programas de magíster de EPA con mención comunitaria/familiar: en torno a esa idea, proyectan tener una primera generación de EPA para el 2018, además de que ya están visualizando el marco legal y ámbito de acción de la EPA, en el contexto de la organización del sistema de salud nacional y las necesidades de la población ${ }^{14}$.

Como se observa, Chile respondió al llamado de la OPS: a nivel nacional organizó un Simposio de EPA para APS (2016), cuyo reporte final describe la contribución de la EPA al sistema de salud, entre la que se señala la atención completa a pacientes crónicos, de modo que al médico solo se remite los casos de pacientes complejos. Lo anterior mejoraría la resolutividad de la APS al ofrecer en una sola prestación cuidados de enfermería y atención médica. En el caso de atención domiciliaria, en una sola prestación la EPA podría entregar cuidados de enfermería y además solicitar y/o tomar exámenes, e iniciar tratamiento o modificar el existente oportunamente, lo cual ahorraría al paciente reiteradas visitas a la APS para atención de salud ${ }^{15}$.

En el caso de Brasil, presenta un gran avance en los posgrados en enfermería respecto de los demás países de Latinoamérica: en el 2014 se lanzó por primera vez una publicación científica en la que se reflexionó en torno al papel de la EPA en América Latina, en forma de editorial de la Revista Brasileña de Enfermería. 
Los autores de dicho estudio recalcan que la EPA puede contribuir al avance de la cobertura universal y del acceso a los servicios de salud, de modo que se ratifica la importancia de los cambios en la formación de la fuerza de trabajo en enfermería. Según ellos, tales cambios deben realizarse a través del desarrollo de políticas que fortalezcan la formación en los niveles de graduación y posgrado, la regulación, el financiamiento, la educación permanente, la práctica profesional y la remuneración. De igual modo, consideraron importante fortalecer la enfermería como categoría profesional, al asumir un papel de protagonista en los procesos de transformación de los sistemas de salud ${ }^{16}$.

En relación con los países de América Latina y el Caribe, Brasil presenta una posición destacada por presentar más condiciones de establecer programas de postgrado en EPA, pues ya posee 51 programas de maestría académica, 15 de maestría profesional y 36 de doctorado en enfermería ${ }^{6}$.

Actualmente, en muchos países de América Latina, las habilidades clínicas necesarias por práctica avanzada se obtienen en el puesto de trabajo en respuesta a las necesidades sanitarias de la población servida, en vez de un programa formal de EPA; por ende, la competencia de EPA y las oportunidades para educación permanente deben ser acreditadas y gestionadas por la facultad de enfermería a nivel universitario ${ }^{3}$.

Los beneficios de la Práctica Avanzada en Enfermería serían inexistentes si no se establece de antemano una buena colaboración y comunicación con otros profesionales de enfermería y con otras disciplinas, para lo cual es esencial definir y evaluar bien el papel de las EPA, así como realizar una previa declaración de intenciones por parte de los miembros del equipo ${ }^{2}$ para evitar malos entendidos.

\section{Marcos para implementar el rol de la EPA}

Existen diferentes modelos para implementar el rol de Enfermería de Práctica Avanzada: el más utilizado es el Marco PEPPA, el cual es un proceso participativo, basado en la evidencia y enfocado en el paciente para el desarrollo, implementación y evaluación de roles de Enfermería de Práctica Avanzada (EPA) ${ }^{17}$.

El marco ha sido utilizado internacionalmente en al menos 16 países y por diversas partes interesadas para presentar los roles de EPA, como es el caso de Canadá. El marco aplica principios de acción participativa que son consistentes con los enfoques recomendados para la planificación de recursos humanos de salud ${ }^{11}$. Para establecer la necesidad del nuevo rol, especifica los siguientes pasos: definir el papel; evaluar la seguridad, eficacia y eficiencia económica de la función; determinar el impacto de la función en la calidad de la atención, la aceptación y la satisfacción del paciente; evaluar la satisfacción del proveedor de atención médica; determinar el grado de transferencia de roles si otras funciones del proveedor de cuidado son asumidas por el rol; y supervisar el rendimiento a largo plazo ${ }^{17}$.

El proceso necesario para la implementación exitosa de los roles de la EPA es tan complejo y dinámico como los roles mismos. El marco PEPPA articula pasos y estrategias para la implementación de roles que son relevantes para las EPA y sus entornos de trabajo; por consiguiente, involucrar a las partes interesadas en el proceso brinda oportunidades para establecer la necesidad e identificar metas compartidas para un rol claramente definido de EPA $^{11}$. 


\section{CONCLUSIONES}

En conclusión, se conoce que actualmente OPS está estableciendo un plan de trabajo para apoyar la ampliación y profesionalización de enfermería de práctica avanzada, mediante el que se identificará objetivos relacionados con educación, reglamento y alcance de la práctica del rol de EPA, y establecerá prioridades para presentar a los estados miembros. Además, OPS procura coordinar una serie de webinars para promover el debate del rol de EPA en América Latina y familiarizar a los docentes de enfermería en América Latina con las condiciones de práctica en otras regiones ${ }^{3}$.

La experiencia internacional señala que la práctica debe preceder a la política; es decir, primero se debe levantar evidencia para luego normar la práctica.

Respecto del ámbito de acción de la EPA, se debe definir su rol, responsabilidades y discutir el nivel de complejidad de la atención de salud que otorgue ${ }^{14}$. Además, uno de los trabajos más grandes para alcanzar este objetivo es que las facultades y escuelas de enfermería incluyan una revisión curricular y preparación docente para dictar nivel avanzado de enfermería en la APS.

Finalmente, este proceso de implementación de la EPA debería estar precedido de un adecuado análisis de la situación, y una planificación para la enfermería de cara a las próximas décadas, así como de un adecuado marco legislativo, dado que un amplio consenso entre las diferentes partes es imprescindible. Por último, el aprovechar la experiencia de otros países y solicitar ayuda a expertos internacionales podría contribuir positivamente al desarrollo de una política basada en la evidencia ${ }^{18}$, ya que un sistema de salud basado en la APS exige compromiso y voluntad políticos a lo largo del tiempo, lo cual requiere mecanismos explícitos que garanticen la sostenibilidad de la $\mathrm{APS}^{4}$.

Mediante la priorización de la preparación y profesionalización de enfermeras de práctica avanzada en América Latina, estarán bien adecuadas para fomentar efectivamente e implementar ampliamente la Cobertura Universal de Salud, y brindar atención primaria de salud de alta calidad donde más se necesita. Debido a que las enfermeras poseen una perspectiva única que prioriza atención holística centrada en la persona que es coherente con el modelo de cuidados de enfermería. Hay que alentar una identidad compartida y una cultura común entre enfermeras de América Latina para apoyar la estandarización del futuro rol de EPA ${ }^{13}$.

Los servicios de salud desempeñan un papel clave en la materialización de muchos de los valores, principios y elementos esenciales de un sistema de salud basado en la APS. Deben ser la puerta de entrada al sistema de atención de salud y han de estar lo más cerca posible de donde la gente vive, trabaja o estudia. Es por ello que los recursos humanos adecuados incluyen a proveedores, trabajadores comunitarios, gestores y personal de apoyo. Deben poseer los conocimientos y habilidades adecuados, además de observar las normas éticas y de tratar a todas las personas con dignidad y respeto4. Aquí es donde entraría el papel que puede llegar a desarrollar una Enfermera de Práctica Avanzada en la Atención Primaria de la Salud. 


\section{REFERENCIAS BIBLIOGRÁFICAS}

1. Paho.org [Internet] Washington, D.C: Organización Panamericana de la Salud/Organización Mundial de la Salud (OPS/OMS), Oficial para las Américas. 2016. Disponible en:

http://www.paho.org/hq/index.php?option=com content\&view=article\&id=12003\%3 Apahowhourges-transformation-of-nursing-education-in-the-americas\&Itemid=135\&lang=es

2. Portillo M. Diferencias entre perfiles enfermeros: práctica avanzada, enfermería asistencia y gestión [monograph on the internet]. [place unknow]: Facultad de Enfermería, Universidad de Navarra; 2010. Disponible en: http://dadun.unav.edu/bitstream/10171/37277/1/Mari\%20Carmen\%20Portillo.pdf

3. Cassiani SHB, Zug KE. Fomentando el papel de la Práctica Avanzada de Enfermería en América Latina. Rev Bras Enferm. 2014; 67 (5):677-8. DOI: http://dx.doi.org/10.1590/0034-7167.2014670501

4. Organización Panamericana de la Salud/Organización Mundial de la Salud (OPS/OMS). La renovación de la Atención Primaria de la Salud en la Américas. Washigton, D.C: OPS/OMS; 2007. Disponible en: http://new.paho.org/hq/dmdocuments/2010/Renovacion_Atencion_Primaria_Salud_Americas-OPS.pdf

5. Zug KE, Cassiani SHB, Pulcini J, Bassalobre GA, Aguirre-Boza F, Park J. Enfermería de práctica avanzada en Latinoamérica y el Caribe: regulación, educación y práctica. Rev. Latino-Am. Enfermagem. 2016; 24: e2807. DOI: http://dx.doi.org/10.1590/1518- 8345.1615.2807.

6. Bryant-Lukosius D, Valaitis R, Martin-Misener R, Donald F, Morán LP, Brousseau L. Enfermería con práctica avanzada: una estrategia para lograr la cobertura universal de salud y el acceso universal a la salud. Rev. Latino-Am. Enfermagem. 2017; 25: e2826. DOI: http://dx.doi.org/10.1590/1518$\underline{8345.1677 .2826}$

7. Dumphy LM, Winland JE, Oscar BP, Thomas DJ. Primary Care in the Twenty-First Cenetury: A Circle of Caring. In: Dumphy LM, Winland JE, Oscar BP, Thomas DJ, editors. Primary Care: The Art and Science of Advanced Practice Nursing. Philadelphia: FA. Davis Company; 2015. p. 3-14.

8. Comellas OM. La construcción de la práctica avanzada en enfermería en el contexto sanitario catalán [Tesis Doctoral]. Barcelona: Escola Universtària d'Infermeria. Universitat de Barcelona. 2015. Disponible en: http://diposit.ub.edu/dspace/bitstream/2445/98504/1/MCO_TESIS.pdf

9. Tracy M, O’Grady E, Hamric A, Hanson C. Advanced Practice Nursing: An Integrative Approach.5th edition. St Louis, Missouri: Saunders; 2014. 
10. Naylor M, Kurtzman E. The role of nurse practitioners in reinventing primary care. Health Aff. [Internet]. 2010; 29(5):893. Disponible en: http://content.healthaffairs.org/content/29/5/893.long

11. Oldenburger D, Cassiani SHB, Bryant-Lokosius D, Valaitis RK, Baumann A, Pulcini J et al. Implementation strategy for advanced practice nursing in primary health care in Latin America and the Caribbean. Rev Panam Salud Pública. 2017; 41. Disponible en: https://scielosp.org/pdf/rpsp/v41/1020-4989-RPSP-41-e40.pdf

12. Nigenda G, Magaña-Valladares L, Cooper K, Ruiz-Larios JA. Recent developments in public health nursing in the Americas. Int J Environ Res Public Health. 2010; 7 (3): 729-750. DOI: http://dx.doi.org/10.3390/ijerph7030729

13. Pan American Health Organization, World Health Organization \& McMaster University. Universal access to health and universal health coverage: Advanced practice nursing summit. [Internet]. Presented at Pan American Health Organization Summit at McMaster University; 1517 April 201;5; Hamilton, Canada. Disponible en: https://www.salud.gob.sv/archivos/enfermeria/PAHO_Advanced_Practice_Nursing_Summit_ Hamilton_CA.pdf

14. Aguirre-Boza F, Achondo B. Atención primaria de salud en Chile: enfermería de práctica avanzada contribuye al acceso universal de salud. Rev. méd. Chile. 2016 Oct; 144 (10): 1319-1321. DOI: http://dx.doi.org/10.4067/S0034-98872016001000011

15. Aguirre Boza F. Enfermería de Práctica Avanzada en la atención primaria: Ahora es el momento. Centros de Estudios Públicos. 2016; 447 Disponible en: https://www.cepchile.cl/cep/site/artic/20161122/asocfile/20161122094316/pder447_faguirre.pdf

16. Miranda Neto MV, Rewa T, Leonello VM, Oliviera MAC. Prática avançada em enfermagem: uma possibilidade para a Atenção Primária em Saúde? Rev Bras Enferm. 2018; 71(supl1):764-9. Disponible en: http://www.scielo.br/pdf/reben/v71s1/pt_0034-7167-reben-71-s1-0716.pdf

17. Bryant-Lukosius D, Spichinger E, Martin J, Stoll H, Kellerhals S, De Geest S, et al. Framework for evaluating the impact of advanced practice nursing roles. J Nurs Scholars. 2016; 48 (2): 201-209. Disponible en: DOI: http://dx.doi.org/10.1111/jnu.12199

18. Galao MR. Enfermería de Práctica Avanzada en España: Ahora es el momento. Index Enferm. 2009 Dic; 18 (4): 221-223. Disponible en: http://scielo.isciii.es/scielo.php?script=sci_arttext\&pid=S1132$12962009000400001 \& \operatorname{lng}=\mathrm{es}$ 